\title{
DETERMINACIÓN DE RIESGO CARDIOVASCULAR EN PERSONAL DE SALUD
}

\section{DETERMINATION OF CARDIOVASCULAR RISK IN HEALTHCARE WORKERS}

\author{
Roberto Islas-Reyes ${ }^{1,2 *}$, Carlos V. Rodríguez-Pérez ${ }^{1,3}$, María F. Muñoz-Lored0 ${ }^{4}$, Ricardo García-Lara ${ }^{5}$ \\ y Joana I. Sámano-Castañeda1,6 \\ 'Departamento de Enseñanza e Investigación, Unidad de Medicina Familiar 47, Instituto Mexicano del Seguro Social; ${ }^{2}$ Escuela de Medicina, \\ Universidad Cuauhtémoc; ${ }^{3}$ Secretaría General, Colegio de la Profesión Médica; ${ }^{4}$ Facultad de Enfermería y Nutrición, Universidad Autónoma de \\ San Luis Potosí; ${ }^{5}$ Hospital de Zona 6, Instituto Mexicano del Seguro Social, Ciudad Valles; ${ }^{6}$ Escuela de Medicina, Universidad del Valle de México. \\ San Luis Potosí, México
}

RESUMEN: Antecedentes: Los estudios de Framingham establecieron el papel de los factores de riesgo en el desarrollo de la cardiopatía isquémica. El tema de la salud de los trabajadores es relevante para los empleadores. Métodos: Estudio observacional transversal para determinar el riesgo cardiovascular en trabajadores de la salud de la Unidad de Medicina Familiar 47 del Instituto Mexicano del Seguro Social por medio de escalas del estudio de Framingham y medidas antropométricas. Universo: 243 individuos. Muestra: 53 individuos de edad $\geq 20$ años. Se realizó cuestionario de riesgo cardiovascular; se tomaron medidas antropométricas, presión arterial sistólica y muestras sanguíneas. Resultados: Edad predominante de 32 a 38 años (32.1\%). Sexo predominante mujeres (67.9\%). Según el puntaje de Framingham, el $94.3 \%$ presenta riesgo bajo, el $3.8 \%$ riesgo moderado y el $1.9 \%$ riesgo alto. Medidas antropométricas: según índice cintura/cadera, el $45.3 \%$ de los participantes no tiene riesgo cardiovascular, el 54.7\% restante sí. Conclusiones: En el personal de salud predomina un riesgo cardiovascular bajo para infarto agudo de miocardio a diez años según el puntaje de Framingham. La mitad de ellos presenta algún grado de sobrepeso u obesidad abdominovisceral. Atenciones preventivas integrales deben ser una medida de diagnóstico oportuno para riesgo cardiovascular.

Palabras clave: Factores de riesgo cardiovascular. Trabajadores de la salud. Presión sanguínea.
ABSTRACT: Background: The Framingham studies established the role of risk factors in the development of ischemic heart disease. The issue of workers' health is quite relevant for employers. Methods: Cross-sectional observational study to determine the cardiovascular risk in health workers of the Family Medicine Unit (FMU) 47 of IMSS by utilizing scales from the Framingham study and anthropometric measures. Universe: 243 individuals. Sample size: 53 individuals 20 years or older. A cardiovascular risk questionnaire was carried out; Anthropometric measurements, systolic blood pressure, and blood samples were taken. Results: Predominant age from 32 to 38 years with $32.1 \%$. Predominantly females with $67.9 \%$. According to the Framingham score, $94.3 \%$ have a low risk, 3.8\% moderate risk, and 1.9\% high risk. Anthropometric measurements: according to the waist/hip ratio, $45.3 \%$ of the participants do not have cardiovascular risk, the remaining 54.7\% have cardiovascular risk. Conclusions: Healthcare workers have a low cardiovascular risk for acute myocardial infarction at ten years, according to, the Framingham score. Half of them have some degree of abdominal overweight or obesity. Comprehensive preventive care should be a timely diagnostic measurement for cardiovascular risk.

Key words: Cardiovascular risk factors. Healthcare workers. Blood pressure.
Correspondencia:

${ }^{*}$ Roberto Islas-Reyes

E-mail: roberto93km@gmail.com
Fecha de recepción: 23-06-2021

Fecha de aceptación: 28-09-2021
Disponible en internet: 03-12-2021 Rev Mex Med Fam. 2021;8:90-6 DOI: 10.24875/RMF.21000061

2007-9710 / C 2021 Federación Mexicana de Especialistas y Residentes en Medicina Familiar. Publicado por Permanyer. Este es un artículo open access bajo la licencia CC BY-NC-ND (http://creativecommons.org/licenses/by-nc-nd/4.0/). 


\section{INTRODUCCIÓN}

Los estudios de Framingham establecieron el papel de los factores de riesgo en el desarrollo de la cardiopatía isquémica, síndrome caracterizado por una disminución del aporte de sangre oxigenada al miocardio ${ }^{1}$.

Existen tablas de predicción de riesgo cardiovascular a diez años basadas en los criterios de Framingham desarrolladas por la Organización Mundial de la Salud (OMS) diseñadas para la zona AMR-B (subregión epidemiológica), que incluye a países de Latinoamérica entre los que se encuentra México. Estas tablas evalúan seis puntos: el sexo, la edad, el consumo de tabaco, presión máxima sistólica en $\mathrm{mmHg}$, diabetes mellitus (DM) y colesterol total (mg/dl), así como colesterol vinculado a lipoproteínas de alta densidad (c-HDL), siendo este último de carácter opcional.

La investigación INTERHEART (estudio de casos y controles sobre factores de riesgo de infarto de miocardio [IAM] en el mundo y América Latina) reportó los factores de riesgo modificables relacionados con eventos cardiovasculares, como tabaco, dislipidemia, DM, hipertensión arterial sistémica y obesidad ${ }^{2}$. Según las guías de práctica clínica del Instituto Mexicano del Seguro Social (IMSS), la enfermedad cardiovascular constituye un grave problema de salud pública mundial por ser la primera causa de morbilidad y mortalidad en varios países ${ }^{3}$. De un total de defunciones por enfermedad cardiovascular, según la OMS, más de tres cuartas partes se producen en los países de ingresos bajos y medios, y desde 1975 la obesidad se ha casi triplicado en todo el mundo. En 2016, más de 1,900 millones de adultos de 18 o más años tenían sobrepeso, de los cuales, más de 650 millones eran obesos 4 . Por otro lado, según datos del Instituto Nacional de Estadística y Geografía (INEGI) 2015 en México, la principal causa de defunción fueron las enfermedades del corazón ${ }^{5}$.

Tomando en cuenta el creciente impacto de las enfermedades cardiovasculares se ha iniciado ya desde hace varios años una oleada de planes de prevención primaria, puesto que varios factores de riesgo para enfermedad cardiovascular son modificables, por lo que es un buen momento para intervenir y causar un impacto positivo a largo plazo.

El tema de la salud de los trabajadores es relevante para los empleadores, esto lo podemos constatar en un artículo de la revista médica HealthAffairs en 2012, el cual aborda el tema del gasto que se destina al cuidado de la salud de los empleados, pues una premisa subyacente de las disposiciones de la Ley del Cuidado de Salud a Bajo Precio alienta a los empleadores a adoptar programas de promoción de la salud, pues hace una asociación entre los riesgos de salud modificables de los trabajadores y el aumento de los costos de atención médica ${ }^{6}$.

Según lo reportado recientemente en un estudio en Ghana, los factores de riesgo cardiometabólico prevalecen entre los proveedores de atención médica ${ }^{7}$. Y en estudios que se han llevado a cabo en nuestro país en trabajadores de la salud se reporta la prevalencia de obesidad y sobrepeso, así como de síndrome metabólico, por lo que se pide tomar acciones inmediatas para modificar el estilo de vida del personal de salud ${ }^{8}$. De igual manera, en diversos países de América Latina y en España se ha reportado la prevalencia de factores de riesgo cardiovascular con el fin de crear conciencia ante las autoridades de que no solamente se debe velar por la salud de los pacientes que acuden a los diversos centros de salud, sino que también hay un deber de ocuparnos por la salud de los trabajadores ${ }^{9,10}$.

Según un artículo del 2020 publicado en la American Journal of Medicine, las enfermedades crónicas se han convertido en la epidemia de nuestros tiempos. Este artículo aborda el tema del modelo de entrega actual y cómo está mal construido para manejar enfermedades crónicas, como lo demuestra la baja adherencia a los indicadores de calidad y el control deficiente de las condiciones tratables ${ }^{11}$. La American Heart Association ha identificado Life's Simple 7 
como los factores más importantes de salud destinados a lograr una salud cardiovascular ideal ${ }^{12}$.

En nuestra unidad clínica de medicina familiar, que es un contacto de primer nivel, laboran un número considerable de trabajadores de la salud y consideramos importante conocer su riesgo cardiovascular a diez años.

En este contexto el objetivo del estudio es determinar el porcentaje de riesgo cardiovascular a diez años mediante el puntaje de Framingham y medidas antropométricas en el personal de salud de la Unidad de Medicina Familiar (UMF) 47 del IMSS San Luis Potosí.

\section{MATERIALY MÉTODOS}

Se realizó un estudio observacional, descriptivo de tipo transversal en personal de salud de la UMF 47 del IMSS en San Luis Potosí, SLP, en el periodo comprendido entre enero y junio del 2021. El muestreo fue aleatorio estratificado con afijación proporcional. Para el cálculo de tamaño de muestra se utilizó el programa Epiinfo diseñado para un estudio descriptivo. El tamaño del universo fue de 243 individuos, con los siguientes parámetros: frecuencia esperada del $50 \%$ y margen de error del 10\%, el tamaño de la muestra fue de 53 individuos.

Se incluyó al personal de salud adscrito a la UMF 47 con edad mayor o igual a 20 años y se excluyó al personal que no se encontraba laborando por incapacidad, personal administrativo, de higiene y limpieza, de conservación y trabajo social. Se eliminaron a los participantes que no contestaron el instrumento completo o que no llenaron el consentimiento informado.

Las variables a recolectar fueron: edad, sexo, tensión arterial sistólica, glucemia en ayunas, colesterol total, tabaquismo, índice cintura/cadera (ICC) y puntaje de Framingham. Para esta última variable se utilizaron los criterios que solicita la calculadora basada en el estudio de Framingham de la página oficial del IMSS exceptuando cifras de HDL de las que se puede prescindir. Se dio a conocer y se explicaron los procedimientos plasmados en el consentimiento informado al personal seleccionado.

Para el procedimiento operacional se aplicó un cuestionario para identificar los siguientes factores de riesgo y antecedentes: edad, sexo, tabaquismo, DM e hipertensión. Posteriormente se realizaron las mediciones antropométricas estandarizadas mediante una cinta metálica antropométrica para uso profesional Lufkin. Se midió el perímetro de la cintura y de la cadera en una posición de pie, con el cuerpo recto y con el abdomen relajado y los pies juntos para obtener el ICC y fueron analizados según los criterios de la OMS. También se tomó la tensión arterial sistólica, la medición se realizó por duplicado utilizando un baumanómetro aneroide, colocado alrededor del brazo e inflando inicialmente por encima de la presión arterial sistólica estimada. Los participantes se mantuvieron en reposo durante al menos diez minutos con la vejiga urinaria vacía, sin haber bebido cafeína, alcohol o haber fumado. Dicho procedimiento en apego estricto a la Guía de Práctica Clínica de diagnóstico y Tratamiento de la Hipertensión Arterial en el Primer nivel de atención. Los instrumentos de medición se calibraron y se mantuvo una observación continua sobre los mismos a fin de evitar sesgos en las tomas. Se aplicó prueba interobservador basada en el método de Bland y Altman con un resultado dentro de los límites del intervalo de concordancia del 95\%.

Finalmente, con el apoyo del departamento de laboratorio se tomaron glucemia en ayunas y colesterol total del personal seleccionado. Se dio a firmar la carta de consentimiento informado. Se procesaron las pruebas en el laboratorio de la unidad.

Los resultados se ordenaron en tablas de Excel de modo que cada dato correspondiera al número asignado del participante, para posteriormente hacer el análisis y obtener el puntaje de acuerdo con la puntuación de Framingham mediante la calculadora basada en el Estudio del Corazón de Framingham del IMSS, la cual fue validada mediante un algoritmo que remeda el uso 
de las clásicas tablas y clasifica el riesgo en tres categorías: bajo $<10 \%$, moderado 10 $20 \%$ y alto $>20 \%$.

Una vez que se obtuvo el resultado del puntaje de Framingham se recolectó junto con las demás variables en tablas de Excel para posteriormente hacer el análisis de los resultados mediante el programa de IBM SPSS.

Este estudio fue aprobado por el comité local de ética en investigación y se realizó según lo establecido en el Reglamento de la Ley General de Salud en Materia de Investigación, la declaración de Helsinki y el informe Belmont, y guiándonos por los principios éticos básicos de respeto, beneficio y justicia.

A los participantes se les proporcionó una carta de consentimiento informado tomando en cuenta los elementos de información precisa, comprensión y voluntariedad.

\section{RESULTADOS}

El rango de edad predominante fue de 32 a 38 años, con un $32.1 \%$ (Fig. 1) y predominaron las mujeres con un total de 36 individuos, lo que representa el $67.9 \%$.

En cuanto a tensión arterial sistólica, el $88.7 \%$ de los participantes obtuvieron cifras normales, un $7.5 \%$ obtuvo cifras que se clasifican como una tensión arterial (TA) normal alta y el $3.8 \%$ obtuvo cifras que están dentro de los rangos de hipertensión arterial grado I. Del 100\% de nuestros participantes el 9.4\% ya tenía antecedente de hipertensión arterial, es decir, que de 53 participantes cinco de ellos ya se conocían con este diagnóstico, sin embargo solo uno de ellos resultó con cifras alteradas durante la medición de la tensión arterial sistólica, los otros cuatro individuos se encontraban con cifras en rangos normales; otros cuatro participantes que no tenían un diagnóstico previo de hipertensión arterial resultaron con cifras alteradas (Fig. 2).

El 3.7\% de los participantes tenían antecedente de DM, sin embargo los valores de glucemia en ayunas arrojaron los siguientes datos: el $77.4 \%$ de la muestra manejó cifras

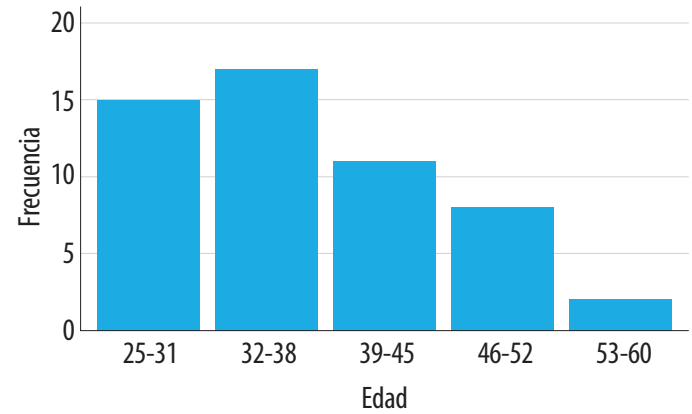

Figura 1. Frecuencia y porcentaje por grupo edad.

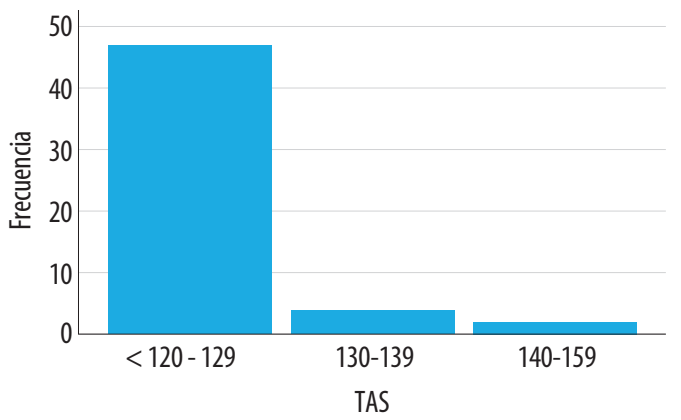

Figura 2. Frecuencia por tensión arterial sistólica (TAS).

normales de glucosa en sangre, el 17\% obtuvo valores alterados $(100-125 \mathrm{mg} / \mathrm{dl})$ y el $5.7 \%$ obtuvo valores que están dentro de los rangos para diagnóstico de DM, es decir, cifras mayores de $126 \mathrm{mg} / \mathrm{dl}^{13}$.

Respecto al colesterol total, el $66 \%$ de la muestra obtuvo cifras de colesterol $<200$, el $24.5 \%$ obtuvo cifras de entre 200-239 y el 9.4\% obtuvo cifras elevadas (<240) (Fig. 4). En cuanto al tema del consumo de tabaco, el $86.8 \%$ refirió no fumar y el $13.2 \%$ dijo que sí.

Según puntaje de Framingham, que evalúa todas las variables anteriormente mencionadas, el 94.3\% de los participantes tiene un riesgo bajo de presentar un IAM a diez años, el 3.8\% tiene un riesgo moderado y el $1.9 \%$ presenta un riesgo alto, estos últimos dos porcentajes que representan el riesgo moderado y alto corresponden a tres individuos y encontramos que su riesgo cardiovascular está ligado a dos factores modificables (glucosa e ICC; [Fig. 3 y Tabla 2]) y uno no modificable (glucosa e ICC) [Tabla 1 y Fig. 3]: edad (comprendida en las 
Tabla 1. Frecuencia y porcentaje del puntaje de la escala de Framingham

\begin{tabular}{|l|l|c|c|}
\hline \multicolumn{2}{|c|}{} & Frecuencia & Porcentaje \\
\hline Puntaje & Bajo & 50 & 94.3 \\
\cline { 2 - 4 } & Moderado & 2 & 3.8 \\
\cline { 2 - 4 } & Alto & 1 & 1.9 \\
\cline { 2 - 4 } & Total & 53 & 100.0 \\
\hline
\end{tabular}

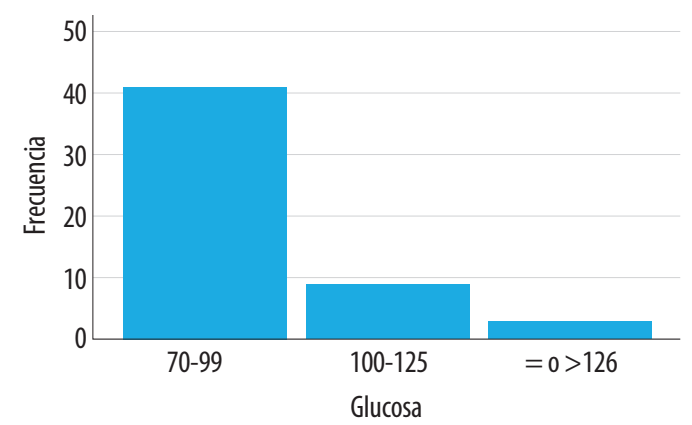

Figura 3. Frecuencia de cifras de glucosa central en ayunas.

categorizaciones 4 [46-52 años] y 5 [53-60 años]).

En cuanto a las medidas antropométricas según el ICC, el $45.3 \%$ de los participantes no tiene riesgo cardiovascular, el $54.7 \%$ restante sí tiene (Tabla 2).

\section{DISCUSIÓN}

En cuanto a las características sociodemográficas de la población estudiada, se encuentra que la media de edad es similar a la reportada por otros autores que han realizado estudios sobre factores de riesgo cardiovascular en personal de salud.

Según lo reportado por Rodríguez, et al. (2016), quienes realizaron su estudio en el IMSS sobre detección de riesgo cardiovascular en 350 trabajadores de la salud, el ICC alterado predominó en el sexo femenino ${ }^{14}$, en nuestros resultados observamos que 17 participantes tuvieron resultados de ICC alterados representando el $54.7 \%$ de nuestra muestra, de los cuales nueve eran mujeres y ocho eran hombres.

Según el mismo estudio de Rodríguez, et al., la TA alterada predominó en el sexo
Tabla 2. Frecuencia y porcentaje de medidas antropométricas

\begin{tabular}{|l|l|c|c|}
\hline \multicolumn{2}{|c|}{} & Frecuencia & Porcentaje \\
\hline \multirow{3}{*}{ IMC } & Sin riesgo & 24 & 45.3 \\
\cline { 2 - 4 } & Con riesgo & 29 & 54.7 \\
\cline { 2 - 4 } & Total & 53 & 100.0 \\
\hline
\end{tabular}

Índice cadera-cintura.

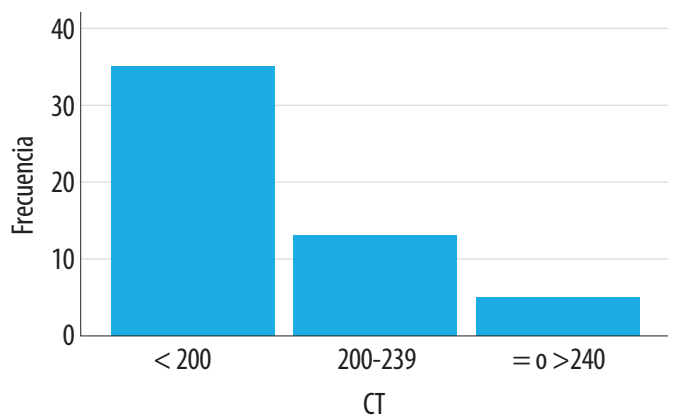

Figura 4. Frecuencia de cifras de colesterol total. CT: colesterol total.

masculino ${ }^{14}$, en nuestro estudio nosotros encontramos que el $9.4 \%$ de nuestra muestra tiene presión arterial sistólica alterada, de los cuales tres son mujeres y dos son hombres. Ellos en su investigación reportan que el 50\% de la muestra presentó glucosa capilar alterada ${ }^{14}$, pero a diferencia de ellos nosotros tomamos glucosa central en ayunas y el $22.7 \%$ de nuestra muestra presentó valores de glucosa alterados, de los cuales $17 \%$ estaban entre 100 y $125 \mathrm{mg} / \mathrm{dl}$ y el 5.7\% tenían valores mayores a $126 \mathrm{mg} / \mathrm{dl}$.

Ondicho, et al. (2016) realizaron un estudio transversal que involucró a 165 personas. Ellos encontraron que la prevalencia de sobrepeso y obesidad fue del $58.8 \%{ }^{15}$, en el estudio actual, mediante los resultados del ICC encontramos que el 32\% tiene cifras alteradas que nos hablan de sobrepeso y obesidad.

Por otro lado Orozco, et al. (2016) estudiaron a 1,089 trabajadores en hospitales de enseñanza de tercer nivel de atención del IMSS y la prevalencia de hipertensión fue del $19 \%{ }^{16}$, en nuestro estudio el $9.4 \%$ 
ya tenía antecedente de hipertensión arterial y el $11.3 \%$ resultó con cifras alteradas, un $7.5 \%$ obtuvo cifras que se clasifican como normal alta y el $3.8 \%$ obtuvo cifras que están dentro de los rangos de hipertensión arterial grado I.

En el mismo estudio de Orozco (2016) se encontró una prevalencia de DM del $9.6 \%{ }^{16}$. En nuestro estudio encontramos que el $3.7 \%$ de los participantes se conocía con antecedente de DM, sin embargo el 5.7\% de nuestra muestra obtuvo resultados de glucosa en ayuno con cifras mayores de $126 \mathrm{mg} / \mathrm{dl}$. Hablando de tema de dislipidemia, Orozco encontró una prevalencia de dislipidemia del 78\%, nosotros encontramos que el 9.4\% de nuestra muestra obtuvo resultados de colesterol total mayores de 240, lo que se clasifica como alto. El mismo estudio de Orozco encontró una prevalencia de tabaquismo del 19\% y nosotros un 13.2\%. Finalmente Orozco, et al. encontraron una prevalencia de sobrepeso y obesidad del 73\%, nosotros encontramos que el $54.7 \%$ de nuestra muestra obtuvo resultados alterados en cuanto a las medidas antropométricas de ICC.

En el presente estudio de investigación observamos que a pesar de obtener valores de riesgo bajo en cuanto al puntaje de Framingham en nuestros participantes los valores de ICC nos arrojaban resultados alterados que nos hablan de un aumento considerable de tejido adiposo abdominovisceral y un riesgo cardiovascular presente. Aunque estas medidas antropométricas solo nos dan la oportunidad de conocer si está o no presente el riesgo, el puntaje de Framingham nos propone varios escalones de riesgo según el porcentaje obtenido de un número determinado de mediciones de laboratorio y antecedentes patológicos de los individuos. A pesar de que el tamaño de la muestra fue pequeño, consideramos que fue suficientemente significativo para la realización de este proyecto, lo cual no demerita en ningún momento los resultados obtenidos, el predominio de participantes fueron adultos jóvenes, lo cual pudo haber reducido los factores de riesgo cardiovascular; quizá en un segundo trabajo incrementar el tamaño muestral nos brinde resultados que podamos contrastar con la presente investigación. De igual manera, en un posterior trabajo de investigación deberían agregarse a los criterios las cifras de c-HDL.

\section{CONCLUSIONES}

En el personal de la UMF 47 predomina un riesgo cardiovascular bajo para IAM a diez años según el puntaje de Framingham. La mitad de los trabajadores de la salud de la UMF 47 presenta algún grado de sobrepeso u obesidad abdominovisceral. Se obtuvo más respuesta del sexo femenino y personal adulto joven. Atenciones preventivas integrales piden ser una medida de diagnóstico oportuno para riesgo cardiovascular.

\section{AGRADECIMIENTOS}

A la memoria del Dr. Jorge Reyes Velázquez. Agradecimiento muy especial al Dr. Carlos Vicente Rodríguez Pérez, tutor del presente proyecto de investigación. Mi profundo agradecimiento a todas las autoridades y personal que conforman la UMF 47, por permitirme realizar todo el proceso investigativo dentro de su establecimiento.

\section{FINANCIAMIENTO}

La presente investigación no ha recibido ninguna beca específica de agencias de los sectores públicos, comercial o sin ánimo de lucro.

\section{CONFLICTO DE INTERESES}

Los autores declaran no tener conflicto de intereses.

\section{RESPONSABILIDADESÉTICAS}

Protección de personas y animales. Los autores declaran que para esta investigación no se han realizado experimentos en seres humanos ni en animales. 
Confidencialidad de los datos. Los autores declaran que han seguido los protocolos de su centro de trabajo sobre la publicación de datos de pacientes.

Derecho a la privacidad y consentimiento informado. Los autores han obtenido el consentimiento informado de los pacientes y/o sujetos referidos en el artículo. Este documento obra en poder del autor de correspondencia.

\section{BIBLIOGRAFÍA}

1. Mahmood SS, Levy D, Vasan RS, Wang TJ. The Framingham Heart Study and the epidemiology of cardiovascular disease: a historical perspective. Lancet. 2014;383(9921):999-1008.

2. Rubio AF. Nuevas guías del American College of Cardiology/ American Heart Association Hypertension para el tratamiento de la hipertensión. ¿Un salto en la dirección correcta? Med Interna Mex. 2018;299-03.

3. Álvarez AAC. Prevención secundaria y rehabilitación cardiaca postinfarto del miocardio en el primer nivel de atención [Internet]. Gobierno de México: Consejo de Salubridad General 2008 [citado: 10 de enero de 2020]. Disponible en: http://www. cenetec-difusion.com/CMGPC/SS-152-08/RR.pdf

4. Organización Mundial de la Salud. Obesidad y sobrepeso [Internet]. Organización Mundial de la Salud; 2021 [citado: 6 de 2021]. Disponible en: https://www.who.int/es/news-room/factsheets/detail/obesity-and-overweight

5. Procuraduría Federal del Consumidor. No rompas más tu corazón. Salud cardiovascular [Internet]. Gobierno de México: Procuraduría Federal del Consumidor; 2017 [citado: 10 de enero de 2020]. Disponible en: https://www.gob.mx/profeco/documentos/no-rompas-mas-tu-corazon-salud-cardiovascular?state $=$ published

6. Goetzel RZ, Pei X, Tabrizi MJ, Henke RM, Kowlessar N, Nelson CF, et al. Ten modifiable health risk factors are linked to more than one-fifth of employer-employee health care spending. Health Aff (Millwood). 2012;31(11):2474-84.

7. Osei J, Kye KK, Owiredu William KBA, Lokpo SY, Esson J, Johnson $\mathrm{BB}$, et al. Cardiometabolic risk factors among healthcare workers: A cross-sectional study at the Sefwi-Wiawso Municipa Hospital, Ghana. Biomed Res Int. 2018;2018:8904548.

8. Cruz-Dominguez MP, González-Márquez F, Ayala-López EA, et al. Overweigth, obesity, metabolic syndrome and waist/height index in health staff. Rev Med Inst Mex Seguro Soc. 2015;53 (Suppl: 1):36-41.

9. Puescas P, Cabrera J, Díaz C. Risk Factors for cardiovascular disease, a problem in health workers. Acta Med Per. 2012;29(1):8-9.

10. López AA, Martinez EA, Rodriguez MR, Vicente T, Salvá MT, Ricci I. Prevalencia de factores de riesgo cardiovascular en trabajadores aparentemente sanos. Gac Med Mex. 2012;148:430-7.

11. Milani RV, Lavie CJ. Health care 2020: reengineering health care delivery to combat chronic disease. Am J Med. 2015;128(4):337-43.

12. Sacco RL. The new American Heart Association 2020 goal: achieving ideal cardiovascular health. J Cardiovasc Med (Hagerstown). 2011;12(4):255-7.

13. American Diabetes Association. 2. Classification and diagnosis of diabetes: Standards of Medical Care in diabetes-2021. Diabetes Care. 2021;44(Suppl 1):S15-33

14. Rodríguez-Reyes RR, Navarro-Zarza JE, Tello-Divicino TL, ParraRojas I, Zaragoza-García O, Guzmán-Guzmán IP. Detection of cardiovascular risk in healthcare workers on the basis of WHO/JNC 7/ATP III criteria. Rev Med Inst Mex Seguro Soc. 2017;55(3):300-8.

15. Ondicho Z, Omondi D, Onyango A. Prevalence and socio-demographic factors associated with overweight and obesity among healthcare workers in Kisumu East Sub-County, Kenya. Am J Med Sci. 2016;6(3):66-72.

16. Orozco-González CN, Cortés-Sanabria L, Viera-Franco رل رل Ramírez-Márquez JJ, Cueto-Manzano AM. Prevalence of cardiovascular risk factors in a population of health-care workers. Rev Med Inst Mex Seguro Soc. 2016;54(5):594-601. 